\title{
A Quantitative Method for In-Situ Pump-Beam Metrology in Ultrafast Electron Microscopy
}

\author{
Jialiang Chen,Chris Leightonand David Flannigan
}

Department of Chemical Engineering and Materials Science, University of Minnesota, Minneapolis, Minnesota, United States

Ultrafast electron microscopy (UEM) with in situ femtosecond (fs) photoexcitation capabilities has been used to study local photoinduced structural dynamics in a variety of nanoscale and nanostructured materials [1-3]. For quantitative photoexcitation conditions so one can determine, for example, the precise energy density absorbed by the specimen, one must have a precise and accurate way to determine the pump-laser spot characteristics at the specimen position. This proves challenging owing to the restricted access and confined space of the region between the objective-lens pole pieces, thus typically necessitating the use of ex situ extrapolation methods. For example, a simple method commonly employed is to measure the laser-beam parameters ex situ on the laser table and then extrapolate the properties via calculation along the remaining pathlength and intervening optics to the specimen position in the UEM [4-6]. Such an approach requires detailed knowledge of the properties of the optics as well as precise alignment conditions that in fact cannot be experimentally verified for the final internal elements in the microscope, thus producing significant uncertainty and relegating quantification to rather poor and unsatisfying estimates.

Here, we describe a simple, quantitative method for measuring the pump-laser spot size in situ at the specimen position in UEM that makes use of ablative-hole generation in amorphous carbon films. Using a well-focused pump beam, ablative holes can be readily generated in the films, and the properties (size and shape) can be linked to the laser-spot properties with an empirical model. Analysis of bright-field images of the laser-ablated holes shows both the shape and size qualitatively match the ex-situ measured profiles (Figure 1). Further, we find a monotonic dependence between the ablated hole size and the pump-pulse energy above a threshold value, below which no hole ablation occurs. To describe the observed pulse-energy dependence, we developed an empirical model that relies on a Gaussian beam profile and an apparent threshold fluence, above which the entire carbon film will be ablated (i.e., a hole will be generated). The resulting expression captures the ablated hole diameter in the $x$ and $y$ dimensions ( $D_{h x}$ and $D_{h y}$, respectively), each as a function of the pump-beam pulse energy, $E_{\text {pulse }}$.

$$
D_{h x}=2 \sigma_{x} \cdot \sqrt{2 \ln \frac{E_{\text {pulse }}}{2 \pi \varepsilon \sigma_{x}^{2} f_{T}^{\prime}}}, D_{h y}=D_{h x} \varepsilon
$$

Here, $\sigma$ and $\varepsilon$ are the pump Gaussian-beam standard deviation and ellipticity, respectively, and $f_{T}^{\prime}$ is the apparent threshold fluence. Thus, experimental plots of the imaged hole diameter, $D$, as a function of pumppulse energy, $E_{\text {pulse }}$, can be least-squares fit with the model by floating $\sigma, \varepsilon$, and $f_{T}^{\prime}$. Further, by testing 515 $\mathrm{nm}$ and 343-nm pump-laser light, we find that the method and model appear to be extendable to a variety of pump wavelengths without modification. Indeed, the data sets presented in Figure 1 for the pair of wavelengths tested are both in good agreement with the model, and the beam sizes at the specimen position determined from the curve fitting agree surprisingly well with the commonly-used ex-situbeam-profiler and extrapolation method, as shown in Table 1 (provided, of course, that the ex-situ method is done extremely accurately and precisely). For an additional check on the validity of the method and model, we performed a systematic study of the observed hole diameter, $D$, as a function of the position of the final focusing lens (i.e., the focal-spot 
size and thus the fluence was varied from the optimum focusing condition). As can be seen in Figure 1, the observed behavior of $D$ as the final lens position is varied (and the fluence at the specimen is varied) agrees well with that predicted using the experimentally-determined fitting parameters $\left(\sigma, \varepsilon\right.$, and $\left.f_{T}^{\prime}\right)$ and the Rayleigh length estimated from the ex-situ beam measurements. We anticipate this method will be extremely useful for conducting more precise and accurate quantitative UEM structural-dynamics measurements [7].

(a)

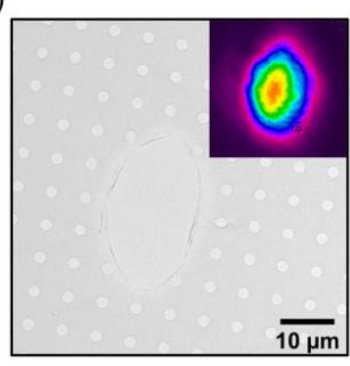

(d)

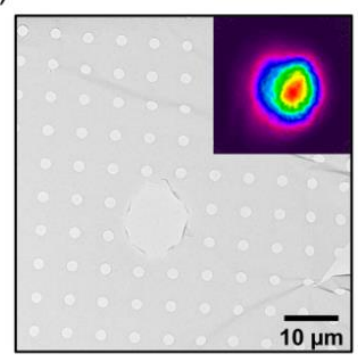

(b)

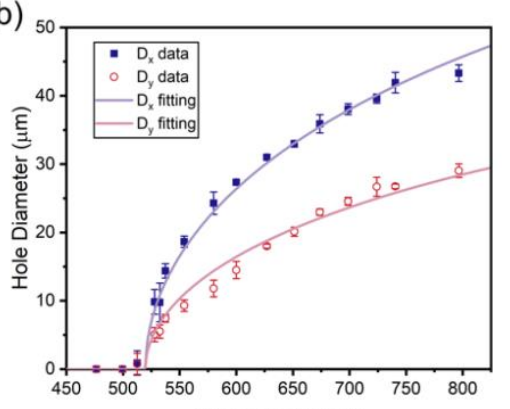

(e)

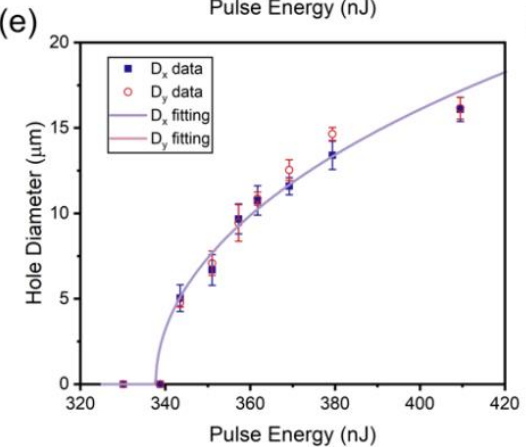

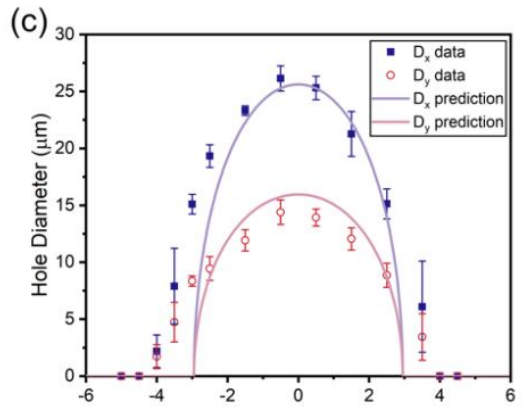

(f)

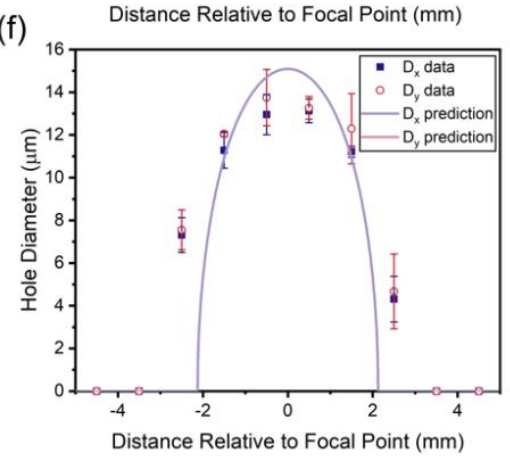

Figure 1. In situ laser ablation experiments on amorphous carbon film using 515-nm (a-c) and 343-nm (d-f) fs laser light. (a,d) Bright-field images of the ablative damage spots. The insets show the corresponding beam profiles measured ex situ with a beam profiler. (b,e) Pulse-energy dependence of the damage spot size. The symbols represent the experimentally determined diameters of the ablation holes created by the fs laser pulse train with varying pulse energies. Solid lines are fits using the empirical damage-hole diameter model described in the text. (c,f) Change in damage-spot size as a function of distance between the final focusing lens and the specimen position. Solid lines are calculated predictions based on the fitting results in (b) and (e) and on the Rayleigh length inferred from the ex-situ measured laser-beam spatial profile.

\begin{tabular}{ccccc}
\hline & Estimated $D_{\mathbf{x}}(\boldsymbol{\mu m})$ & Measured $\mathbf{D}_{\mathbf{x}}(\boldsymbol{\mu m})$ & Estimated $\mathbf{D}_{\mathbf{y}}(\boldsymbol{\mu m})$ & Measured $\mathbf{D}_{\mathbf{y}}(\boldsymbol{\mu m})$ \\
\hline $\mathbf{3 4 3 - n m}$ & 52.5 & $55.3 \pm 0.8$ & 48.6 & $55.3 \pm 2.4$ \\
$\mathbf{5 1 5}-\mathbf{n m}$ & 87.8 & $98.5 \pm 0.5$ & 65.6 & $61.0 \pm 1.0$ \\
\hline
\end{tabular}

Figure 2. Laser-beam size extrapolated to the specimen position from the ex-situ measured properties compared to the laser-beam size measured using the in-situ ablative method presented here. Beam diameter is defined as the e-2 value.

\section{References}

[1] AH Zewail, Science 328 (2010), p. 187.

[2] DJ Flannigan and AH Zewail, Acc. Chem. Res. 45 (2012), p. 1828.

[3] DA Plemmons, PK Suri, and DJ Flannigan, Chem. Mater. 27 (2015), p. 3178.

[4] G Cao, et al., Sci. Rep. 5 (2015), p. 8404.

[5] L Piazza, et al., Chem. Phys. 423 (2013), p. 79. 
[6] KB Schliep, et al., Appl. Phys. Lett. 110 (2017), p. 222404.

[7]This work was supported partially by the American Chemical Society Petroleum Research Fund and partially by the U.S. Department of Energy through the UMN Center for Quantum Materials under Grant No. DE-SC-0016371. 